Geometry $\& \mathcal{T}$ Topology

Volume 6 (2002) 409-424

Published: 15 September 2002

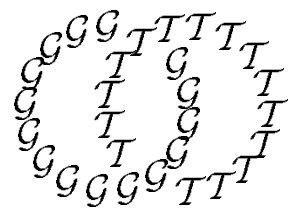

\title{
On the Cut Number of a 3-manifold
}

\author{
SHelly L HARVEY \\ Department of Mathematics \\ University of California at San Diego \\ La Jolla, CA 92093-0112, USA \\ Email: sharvey@math.ucsd.edu \\ URL: http://math.ucsd.edu/ `sharvey
}

\begin{abstract}
The question was raised as to whether the cut number of a $3-$ manifold $X$ is bounded from below by $\frac{1}{3} \beta_{1}(X)$. We show that the answer to this question is "no." For each $m \geq 1$, we construct explicit examples of closed 3-manifolds $X$ with $\beta_{1}(X)=m$ and cut number 1 . That is, $\pi_{1}(X)$ cannot map onto any non-abelian free group. Moreover, we show that these examples can be assumed to be hyperbolic.
\end{abstract}

AMS Classification numbers Primary: 57M27, 57N10

Secondary: 57M05, 57M50, 20F34, 20F67

Keywords: 3-manifold, fundamental group, corank, Alexander module, virtual betti number, free group

Proposed: Cameron Gordon

Seconded: Joan Birman, Walter Neumann

(C) Geometry $\& \mathcal{G}$ Topology $\mathcal{P}$ ublications
Received: 27 February 2002

Accepted: 22 August 2002 


\section{Introduction}

Let $X$ be a closed, orientable $n$-manifold. The cut number of $X, c(X)$, is defined to be the maximal number of components of a closed, 2-sided, orientable hypersurface $F \subset X$ such that $X-F$ is connected. Hence, for any $n \leq$ $c(X)$, we can construct a map $f: X \rightarrow \bigvee_{i=1}^{n} S^{1}$ such that the induced map on $\pi_{1}$ is surjective. That is, there exists a surjective map $f_{*}: \pi_{1}(X) \rightarrow F(c)$, where $F(c)$ is the free group with $c=c(X)$ generators. Conversely, if we have any epimorphism $\phi: \pi_{1}(X) \rightarrow F(n)$, then we can find a map $f: X \rightarrow$ $\bigvee_{i=1}^{n} S^{1}$ such that $f_{*}=\phi$. After making the $f$ transverse to a non-wedge point $x_{i}$ on each $S^{1}, f^{-1}(X)$ will give $n$ disjoint surfaces $F=\cup F_{i}$ with $X-F$ connected. Hence one has the following elementary group-theoretic characterization of $c(X)$.

Proposition 1.1 $c(X)$ is the maximal $n$ such that there is an epimorphism $\phi: \pi_{1}(X) \rightarrow F(n)$ onto the free group with $n$ generators.

Example 1.2 Let $X=S^{1} \times S^{1} \times S^{1}$ be the 3 -torus. Since $\pi_{1}(X)=\mathbb{Z}^{3}$ is abelian, $c(X)=1$.

Using Proposition 1.1, we show that the cut number is additive under connected sum.

Proposition 1.3 If $X=X_{1} \# X_{2}$ is the connected sum of $X_{1}$ and $X_{2}$ then

$$
c(X)=c\left(X_{1}\right)+c\left(X_{2}\right) .
$$

Proof Let $G_{i}=\pi_{1}\left(X_{i}\right)$ for $i=1,2$ and $G=\pi_{1}(X) \cong G_{1} * G_{2}$. It is clear that $G$ maps surjectively onto $F\left(c\left(X_{1}\right)\right) * F\left(c\left(X_{2}\right)\right) \cong F\left(c\left(X_{1}+X_{2}\right)\right)$. Therefore $c(X) \geq c\left(X_{1}\right)+c\left(X_{2}\right)$.

Now suppose that there exists a map $\phi: G \rightarrow F(n)$. Let $\phi_{i}: G_{i} \rightarrow F(n)$ be the composition $G_{i} \rightarrow G_{1} * G_{2} \cong G \stackrel{\phi}{\rightarrow} F(n)$. Since $\phi$ is surjective and $G \cong G_{1} * G_{2}, \operatorname{Im}\left(\phi_{1}\right)$ and $\operatorname{Im}\left(\phi_{2}\right)$ generate $F(n)$. Morever, $\operatorname{Im}\left(\phi_{i}\right)$ is a subgroup of a free group, hence is free of rank less than or equal to $c\left(X_{i}\right)$. It follows that $n \leq c\left(X_{1}\right)+c\left(X_{2}\right)$. In particular, when $n$ is maximal we have $c(X)=n \leq c\left(X_{1}\right)+c\left(X_{2}\right)$.

In this paper, we will only consider 3 -manifolds with $\beta_{1}(X) \geq 1$. Consider the surjective map $\pi_{1}(X) \rightarrow H_{1}(X) /\{\mathbb{Z}$-torsion $\} \cong \mathbb{Z}^{\beta_{1}(X)}$. Since $\beta_{1}(X) \geq 1$, 
we can find a surjective map from $\mathbb{Z}^{\beta_{1}(X)}$ onto $\mathbb{Z}$. It follows from Proposition 1.1 that $c(X) \geq 1$. Moreover, every map $\phi: \pi_{1}(X) \rightarrow F(n)$ gives rise to an epimorphism $\bar{\phi}: H_{1}(X) \rightarrow H_{1}\left(\bigvee_{i=1}^{n} S^{1}\right) \cong \mathbb{Z}^{n}$ It follows that $\beta_{1}(X) \geq n$ which gives us the well known result:

$$
1 \leq c(X) \leq \beta_{1}(X) .
$$

It has recently been asked whether a (non-trivial) lower bound exists for the cut number. We make the following observations.

Remark 1.4 If $S$ is a closed, orientable surface then $c(S)=\frac{1}{2} \beta_{1}(S)$.

Remark 1.5 If $X$ has solvable fundamental group then $c(X)=1$ and $\beta_{1}(X)$ $\leq 3$.

Remark 1.6 Both $c$ and $\beta_{1}$ are additive under connected sum (Proposition $1.3)$.

Therefore it is natural to ask the following question first asked by A Sikora and $\mathrm{T}$ Kerler. This question was motivated by certain results and conjectures on the divisibility of quantum 3-manifold invariants by P Gilmer-T Kerler [2] and T Cochran-P Melvin [1].

Question 1.7 Is $c(X) \geq \frac{1}{3} \beta_{1}(X)$ for all closed, orientable 3-manifolds $X$ ?

We show that the answer to this question is "as far from yes as possible." In fact, we show that for each $m \geq 1$ there exists a closed, hyperbolic 3-manifold with $\beta_{1}(X)=m$ and $c(X)=1$. We actually prove a stronger statement.

Theorem 3.1 For each $m \geq 1$ there exist closed 3-manifolds $X$ with $\beta_{1}(X)$ $=m$ such that for any infinite cyclic cover $X_{\phi} \rightarrow X, \operatorname{rank}_{\mathbb{Z}\left[t^{ \pm 1}\right]} H_{1}\left(X_{\phi}\right)=0$.

We note the condition stated in the Theorem 3.1 is especially interesting because of the following theorem of J Howie [3]. Recall that a group $G$ is large if some subgroup of finite index has a non-abelian free homomorphic image. Howie shows that if $G$ has an infinite cyclic cover whose rank is at least 1 then $G$ is large.

Theorem 1.8 (Howie [3]) Suppose that $\widetilde{K}$ is a connected regular covering complex of a finite 2-complex $K$, with nontrivial free abelian covering transformation group $A$. Suppose also that $H_{1}(\widetilde{K} ; \mathbb{Q})$ has a free $\mathbb{Q}[A]$-submodule of rank at least 1 . Then $G=\pi_{1}(K)$ is large. 
Using the proof of Theorem 3.1 we show that the fundamental group of the aforementioned 3-manifolds cannot map onto $F / F_{4}$ where $F$ is the free group with 2 generators and $F_{4}$ is the $4^{\text {th }}$ term of the lower central series of $F$.

Proposition 3.3 Let $X$ be as in Theorem 3.1, $G=\pi_{1}(X)$ and $F$ be the free group on 2 generators. There is no epimorphism from $G$ onto $F / F_{4}$.

Independently, A Sikora has recently shown that the cut number of a "generic" 3 -manifold is at most 2 [8]. Also, C Leininger and A Reid have constructed specific examples of genus 2 surface bundles $X$ satisfying (i) $\beta_{1}(X)=5$ and $c(X)=1$ and (ii) $\beta_{1}(X)=7$ and $c(X)=2[6]$.

Acknowledgements I became interested in the question as to whether the cut number of a 3-manifold was bounded below by one-third the first betti number after hearing it asked by A Sikora at a problem session of the 2001 Georgia Topology Conference. The question was also posed in a talk by $\mathrm{T}$ Kerler at the 2001 Lehigh Geometry and Topology Conference. The author was supported by NSF DMS-0104275 as well as by the Bob E and Lore Merten Watt Fellowship.

\section{Relative Cut Number}

Let $\phi$ be a primitive class in $H^{1}(X ; \mathbb{Z})$. Since $H^{1}(X ; \mathbb{Z}) \cong \operatorname{Hom}\left(\pi_{1}(X), \mathbb{Z}\right)$, we can assume $\phi$ is a surjective homomorphism, $\phi: \pi_{1}(X) \rightarrow \mathbb{Z}$. Since $X$ is an orientable $3-$ manifold, every element in $H_{2}(X ; \mathbb{Z})$ can be represented by an embedded, oriented, 2-sided surface [10, Lemma 1]. Therefore, if $\phi \in$ $H^{1}(X ; \mathbb{Z}) \cong H_{2}(X ; \mathbb{Z})$ there exists a surface (not unique) dual to $\phi$. The cut number of $X$ relative to $\phi, c(X, \phi)$, is defined as the maximal number of components of a closed, 2-sided, oriented surface $F \subset X$ such that $X-F$ is connected and one of the components of $F$ is dual to $\phi$. In the above definition, we could have required that "any number" of components of $F$ be dual to $\phi$ as opposed to just "one." We remark that since $X-F$ is connected, these two conditions are equivalent. Similar to $c(X)$, we can describe $c(X, \phi)$ group theoretically.

Proposition 2.1 $c(X, \phi)$ is the maximal $n$ such that there is an epimorphism $\psi: \pi_{1}(X) \rightarrow F(n)$ onto the free group with $n$ generators that factors through $\phi$ (see diagram on next page). 


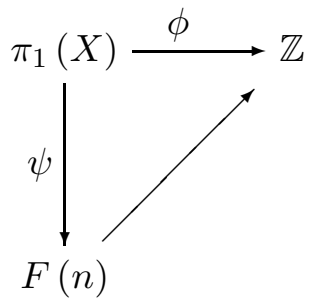

It follows immediately from the definitions that $c(X, \phi) \leq c(X)$ for all primitive $\phi$. Now let $F$ be any surface with $c(X)$ components and let $\phi$ be dual to one of the components, then $c(X, \phi)=c(X)$. Hence

$$
c(X)=\max \left\{c(X, \phi) \mid \phi \text { is a primitive element of } H^{1}(X ; \mathbb{Z})\right\} .
$$

In particular, if $c(X, \phi)=1$ for all $\phi$ then $c(X)=1$.

We wish to find sufficient conditions for $c(X, \phi)=1$. In [5, page 44], T Kerler develops a skein theoretic algorithm to compute the one-variable Alexander polynomial $\Delta_{X, \phi}$ from a surgery presentation of $X$. As a result, he shows that if $c(X, \phi) \geq 2$ then the Frohman-Nicas TQFT evaluated on the cut cobordism is zero, implying that $\Delta_{X, \phi}=0$. Using the fact that $\mathbb{Q}\left[t^{ \pm 1}\right]$ is a principal ideal domain one can prove that $\Delta_{X, \phi}=0$ is equivalent to $\operatorname{rank}_{\mathbb{Z}\left[t^{ \pm 1}\right]} H_{1}\left(X_{\phi}\right) \geq 1$. We give an elementary proof of the equivalent statement of Kerler's.

Proposition 2.2 If $c(X, \phi) \geq 2$ then $\operatorname{rank}_{\mathbb{Z}\left[t^{ \pm 1}\right]} H_{1}\left(X_{\phi}\right) \geq 1$.

Proof Suppose $c(X, \phi) \geq 2$ then there is a surjective map $\psi: \pi_{1}(X) \rightarrow F(n)$ that factors through $\phi$ with $n \geq 2$. Let $\bar{\phi}: F(n) \rightarrow \mathbb{Z}$ be the homomorphism such that $\phi=\bar{\phi} \circ \psi$. $\phi$ surjective implies that $\psi_{\mid \operatorname{ker} \phi}: \operatorname{ker} \phi \rightarrow \operatorname{ker} \bar{\phi}$ is surjective. Writing $\mathbb{Z}$ as the multiplicative group generated by $t$, we can consider $\frac{\operatorname{ker} \phi}{[\operatorname{ker} \phi, \operatorname{ker} \phi]}$ and $\frac{\operatorname{ker} \bar{\phi}}{[\operatorname{ker} \bar{\phi}, \operatorname{ker} \bar{\phi}]}$ as modules over $\mathbb{Z}\left[t^{ \pm 1}\right]$. Here, the $\mathrm{t}$ acts by conjugating by an element that maps to t by $\phi$ or $\bar{\phi}$. Moreover, $\psi_{\mid \operatorname{ker} \phi}: \frac{\operatorname{ker} \phi}{[\operatorname{ker} \phi, \operatorname{ker} \phi]} \rightarrow$ $\frac{\operatorname{ker} \bar{\phi}}{\operatorname{ker} \bar{\phi}, \operatorname{ker} \bar{\phi}]}$ is surjective hence

$$
\operatorname{rank}_{\mathbb{Z}\left[t^{ \pm 1}\right]}\left(\frac{\operatorname{ker} \phi}{[\operatorname{ker} \phi, \operatorname{ker} \phi]}\right) \geq \operatorname{rank}_{\mathbb{Z}\left[t^{ \pm 1}\right]}\left(\frac{\operatorname{ker} \bar{\phi}}{[\operatorname{ker} \bar{\phi}, \operatorname{ker} \bar{\phi}]}\right)=n-1 .
$$

Since $n \geq 2, \operatorname{rank}_{\mathbb{Z}\left[t^{ \pm 1}\right]} H_{1}\left(X_{\phi}\right)=\operatorname{rank}_{\mathbb{Z}\left[t^{ \pm 1}\right]}\left(\frac{\operatorname{ker} \phi}{[\operatorname{ker} \phi, \operatorname{ker} \phi]}\right) \geq 1$.

Corollary 2.3 If $\pi_{1}(X) \rightarrow F / F^{\prime \prime}$ where $F$ is a free group of rank 2 then there exists a $\phi: \pi_{1}(X) \rightarrow \mathbb{Z}$ such that $\operatorname{rank}_{\mathbb{Z}\left[t^{ \pm 1}\right]} H_{1}\left(X_{\phi}\right) \geq 1$. 
Proof This follows immediately from the proof of Proposition 2.2 after noticing that $F^{\prime \prime} \subset[\operatorname{ker}(\bar{\phi}), \operatorname{ker}(\bar{\phi})]$ and $\operatorname{Hom}\left(F / F^{\prime \prime}, \mathbb{Z}\right) \cong \operatorname{Hom}(F, \mathbb{Z})$.

\section{The Examples}

We construct closed 3-manifolds all of whose infinite cyclic covers have first homology that is $\mathbb{Z}\left[t^{ \pm 1}\right]$-torsion. The 3 -manifolds we consider are 0 -surgery on an $m$-component link that is obtained from the trivial link by tying a Whitehead link interaction between each two components.

Theorem 3.1 For each $m \geq 1$ there exist closed 3-manifolds $X$ with $\beta_{1}(X)$ $=m$ such that for any infinite cyclic cover $X_{\phi} \rightarrow X, \operatorname{rank}_{\mathbb{Z}\left[t^{ \pm 1}\right]} H_{1}\left(X_{\phi}\right)=0$.

It follows from Propostion 2.2 that the cut number of the manifolds in Theorem 3.1 is 1 . In fact, Corollary 2.3 implies that $\pi_{1}(X)$ does not map onto $F / F^{\prime \prime}$ where $F$ is a free group of rank 2. Moreover, the proof of this theorem shows that $\pi_{1}(X)$ does not even map onto $F / F_{4}$ where $F_{n}$ is the $n^{\text {th }}$ term of the lower central series of $F$ (see Proposition 3.3).

By a theorem of Ruberman [7], we can assume that the manifolds with cut number 1 are hyperbolic.

Corollary 3.2 For each $m \geq 1$ there exist closed, orientable, hyperbolic 3manifolds $Y$ with $\beta_{1}(Y)=m$ such that for any infinite cyclic cover $Y_{\phi} \rightarrow Y$, $\operatorname{rank}_{\mathbb{Z}\left[t^{ \pm 1}\right]} H_{1}\left(Y_{\phi}\right)=0$.

Proof Let $X$ be one of the 3-manifolds in Theorem 3.1. By [7, Theorem 2.6], there exists a degree one map $f: Y \rightarrow X$ where $Y$ is hyperbolic and $f_{*}$ is an isomorphism on $H_{*}$. Denote by $G=\pi_{1}(X)$ and $P=\pi_{1}(Y)$. It is then well-known that $f$ is surjective on $\pi_{1}$. It follows from Stalling's theorem $[9$, page 170] that the kernel of $f_{*}$ is $P_{\omega} \equiv \cap P_{n}$. Now, suppose $\phi: P \stackrel{f_{*}}{\rightarrow} G \stackrel{\bar{\phi}}{\rightarrow} \mathbb{Z}$ defines an infinite cyclic cover of $Y$. Then $H_{1}\left(Y_{\phi}\right) \rightarrow H_{1}\left(X_{\bar{\phi}}\right)$ has kernel $P_{\omega} /[\operatorname{ker} \phi, \operatorname{ker} \phi]$. To show that $\operatorname{rank}_{\mathbb{Z}\left[t^{ \pm 1}\right]} H_{1}\left(Y_{\phi}\right)=0$ it suffices to show that $P_{\omega}$ vanishes under the map $H_{1}\left(Y_{\phi}\right) \rightarrow H_{1}\left(Y_{\phi}\right) \otimes_{\mathbb{Z}\left[t^{ \pm 1}\right]} \mathbb{Q}\left[t^{ \pm 1}\right] \rightarrow H_{1}\left(Y_{\phi}\right) \otimes_{\mathbb{Z}\left[t^{ \pm 1}\right]}$ $\mathbb{Q}(t)$ since then $\operatorname{rank}_{\mathbb{Z}\left[t^{ \pm 1}\right]} H_{1}\left(Y_{\phi}\right)=\operatorname{rank}_{\mathbb{Z}\left[t^{ \pm 1}\right]} H_{1}\left(X_{\bar{\phi}}\right)=0$.

Note that $H_{1}\left(Y_{\phi}\right) \otimes_{\mathbb{Z}\left[t^{ \pm 1}\right]} \mathbb{Q}\left[t^{ \pm 1}\right] \cong \bigoplus_{i=1}^{n} \mathbb{Q}\left[t^{ \pm 1}\right] \oplus T$ where $T$ is a $\mathbb{Q}\left[t^{ \pm 1}\right]$ torsion module. Moreover, $P_{n}$ is generated by elements of the form $\gamma=$ 
$\left[p_{1}\left[p_{2}\left[p_{3}, \ldots\left[p_{n-2}, \alpha\right]\right]\right]\right]$ where $\alpha \in P_{2} \subseteq \operatorname{ker} \phi$. Therefore

$$
[\gamma]=\left(\phi\left(p_{i}\right)-1\right) \cdots\left(\phi\left(p_{n-2}\right)-1\right)[\alpha]
$$

in $H_{1}\left(Y_{\phi}\right)$ which implies that $P_{n} \subseteq J^{n-2}\left(H_{1}\left(Y_{\phi}\right)\right)$ for $n \geq 2$ where $J$ is the augmentation ideal of $\mathbb{Z}\left[t^{ \pm 1}\right]$. It follows that any element of $P_{\omega}$ considered as an element of $H_{1}\left(Y_{\phi}\right) \otimes_{\mathbb{Z}\left[t^{ \pm 1}\right]} \mathbb{Q}\left[t^{ \pm 1}\right]$ is infinitely divisible by $t-1$ and hence is torsion.

Proof of Theorem 3.1 Let $L=\sqcup L_{i}$ be the oriented trivial link with $m$ components in $S^{3}$ and $\sqcup D_{i}$ be oriented disjoint disks with $\partial D_{i}=L_{i}$. The fundamental group of $S^{3}-L$ is freely generated by $\left\{x_{i}\right\}$ where $x_{i}$ is a meridian curve of $L_{i}$ which intersects $D_{i}$ exactly once and $D_{i} \cdot x_{i}=1$. For all $i, j$ with $1 \leq i<j \leq m$ let $\alpha_{i j}: I \rightarrow S^{3}$ be oriented disjointly embedded arcs such that $\alpha_{i j}(0) \in L_{i}$ and $\alpha_{i j}(1) \in L_{j}$ and $\alpha_{i j}(I)$ does not intersect $\sqcup D_{i}$. For each arc $\alpha_{i j}$, let $\gamma_{i j}$ be the curve embedded in a small neighborhood of $\alpha_{i j}$ representing the class $\left[x_{i}, x_{j}\right]$ as in Figure 1 . Let $X$ be the 3 -manifold obtained performing
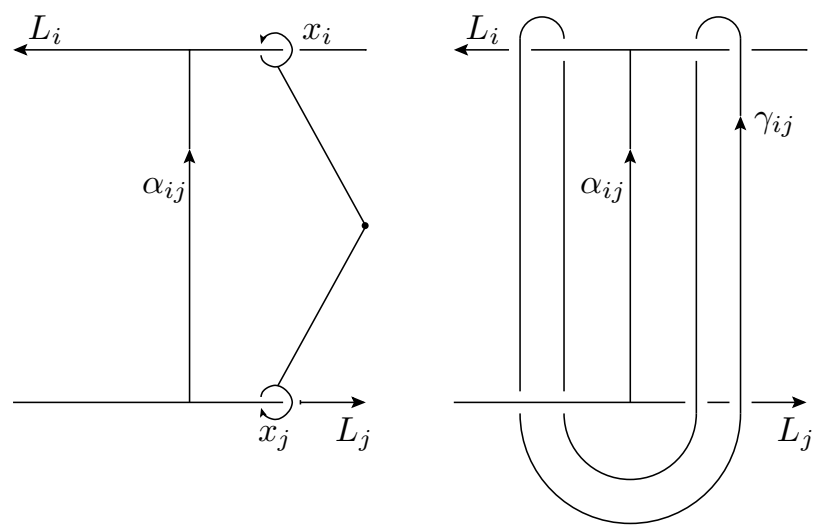

Figure 1

0-framed Dehn surgery on $L$ and -1-framed Dehn surgery on each $\gamma=\sqcup \gamma_{i j}$. See Figure 2 for an example of $X$ when $m=5$.

Denote by $X_{0}$, the manifold obtained by performing 0-framed Dehn surgery on $L$. Let $W$ be the 4 -manifold obtained by adding a 2 -handle to $X_{0} \times I$ along each curve $\gamma_{i j} \times\{1\}$ with framing coefficient -1 . The boundary of $W$ is $\partial W=X_{0} \sqcup-X$. We note that

$$
\left.\pi_{1}(W)=\left\langle x_{1}, \ldots, x_{m}\right|\left[x_{i}, x_{j}\right]=1 \text { for all } 1 \leq i<j \leq m\right\rangle \cong \mathbb{Z}^{m} .
$$




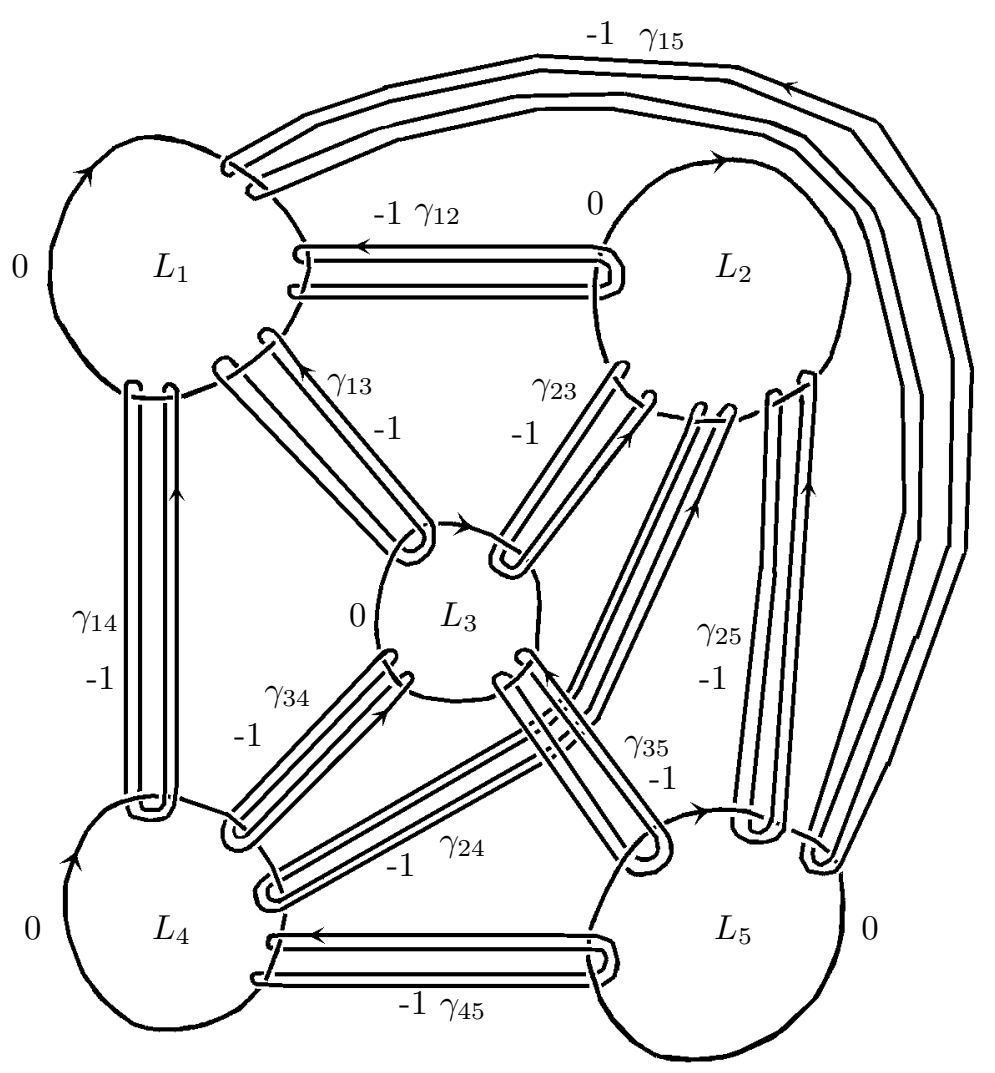

Figure 2: The surgered manifold $X$ when $m=5$

Let $\left\{x_{i k}, \mu_{i j l}\right\}$ be the generators of $\pi_{1}\left(S^{3}-(L \sqcup \gamma)\right)$ that are obtained from a Wirtinger presentation where $x_{i k}$ are meridians of the $i^{\text {th }}$ component of $L$ and $\mu_{i j l}$ are meridians of the $(i, j)^{t h}$ component of $\gamma$. Note that $\left\{x_{i k}, \mu_{i j l}\right\}$ generate $G \equiv \pi_{1}(X)$. For each $1 \leq i \leq m$ let $\bar{x}_{i}=x_{i 1}$ and $\bar{\mu}_{i j}$ be the specific $\mu_{i j l}$ that is denoted in Figure 3. We will use the convention that

$$
[a, b]=a b a^{-1} b^{-1}
$$

and

$$
a^{b}=b a b^{-1}
$$

We can choose a projection of the trivial link so that the arcs $\alpha_{i j}$ do not pass under a component of $L$. Since $\bar{\mu}_{i j}$ is equal to a longitude of the curve $\gamma_{i j}$ in $X$, we have $\bar{\mu}_{i j}=\left[x_{i n_{i j}}, \lambda x_{j n_{j i}} \lambda^{-1}\right]$ for some $n_{i j}$ and $n_{j i}$ and $\lambda$ where $\lambda$ is a product of conjugates of meridian curves $\bar{\mu}_{l k}$ and $\bar{\mu}_{l k}^{-1}$. Moreover, we can find 


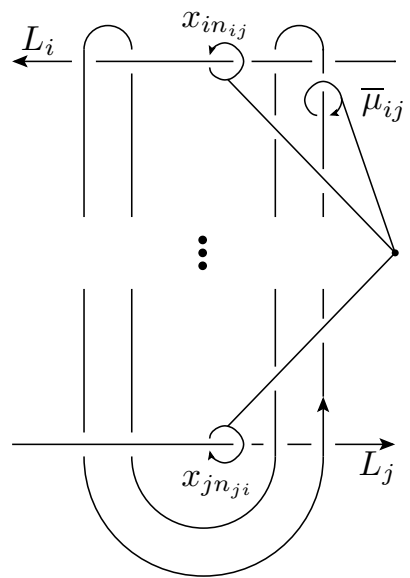

Figure 3

a projection of $L \sqcup \gamma$ so that the individual components of $L$ do not pass under or over one another. Hence $x_{i j}=\omega \bar{x}_{i} \omega^{-1}$ where $\omega$ is a product of conjugates of the meridian curves $\bar{\mu}_{l k}$ and $\bar{\mu}_{l k}^{-1}$. As a result, we have

$$
\begin{aligned}
\bar{\mu}_{i j} & =\left[x_{i n_{i j}}, \lambda x_{j n_{j i}} \lambda^{-1}\right] \\
& =\left[\omega_{1} \bar{x}_{i} \omega_{1}^{-1}, \lambda \omega_{2} \bar{x}_{j} \omega_{2}^{-1} \lambda^{-1}\right] \\
& =\left[\bar{x}_{i}, \omega_{1}^{-1} \lambda \omega_{2} \bar{x}_{j} \omega_{2}^{-1} \lambda^{-1} \omega_{1}^{-1}\right]^{\omega_{1}}
\end{aligned}
$$

for some $\lambda, \omega_{1}$, and $\omega_{2}$.

We note that $\bar{\mu}_{i j}=\left[x_{i n_{i j}}, \lambda x_{j n_{j i}} \lambda^{-1}\right]$ hence $\bar{\mu}_{i j} \in G^{\prime}$ for all $i<j$. Setting $v=\omega_{1}^{-1} \lambda \omega_{2}$ and using the equality

$$
[a, b c]=[a, b][a, c]^{b}
$$

we see that

$$
\begin{aligned}
\bar{\mu}_{i j} & =\left[\bar{x}_{i}, v \bar{x}_{j} v^{-1}\right]^{\omega_{1}} \\
& =\left[\bar{x}_{i}, v \bar{x}_{j} v^{-1}\right] \bmod G^{\prime \prime} \\
& =\left[\bar{x}_{i},\left[v, \bar{x}_{j}\right] \bar{x}_{j}\right] \\
& =\left[\bar{x}_{i},\left[v, \bar{x}_{j}\right]\right]\left[\bar{x}_{i}, \bar{x}_{j}\right]^{\left[v, \bar{x}_{j}\right]} \\
& =\left[\bar{x}_{i},\left[v, \bar{x}_{j}\right]\right]\left[\bar{x}_{i}, \bar{x}_{j}\right] \bmod G^{\prime \prime}
\end{aligned}
$$

since $\omega_{1}, v \in G^{\prime}$.

Consider the dual relative handlebody decomposition $(W, X)$. $W$ can be obtained from $X$ by adding a 0 -framed 2-handle to $X \times I$ along each of the 
meridian curves $\bar{\mu}_{i j} \times\{1\}$. (3) implies that $\bar{\mu}_{i j}$ is trivial in $H_{1}(X)$ hence the inclusion map $j: X \rightarrow W$ induces an isomorphism $j_{*}: H_{1}(X) \cong H_{1}(W)$. Therefore if $\phi: G \rightarrow \Lambda$ where $\Lambda$ is abelian then there exists a $\psi: \pi_{1}(W) \rightarrow \Lambda$ such that $\psi \circ j_{*}=\phi$.

Suppose $\phi: G \rightarrow\langle t\rangle \cong \mathbb{Z}$ and $\psi: \pi_{1}(W) \rightarrow\langle t\rangle$ is an extension of $\phi$ to $\pi_{1}(W)$. Let $X_{\phi}$ and $W_{\psi}$ be the infinite cyclic covers of $W$ and $X$ corresponding to $\psi$ and $\phi$ respectively. Consider the long exact sequence of pairs,

$$
\rightarrow H_{2}\left(W_{\psi}, X_{\phi}\right) \stackrel{\partial_{*}}{\rightarrow} H_{1}\left(X_{\phi}\right) \rightarrow H_{1}\left(W_{\psi}\right) \rightarrow
$$

Since $\pi_{1}(W) \cong \mathbb{Z}^{m}, H_{1}\left(W_{\psi}\right) \cong \mathbb{Z}^{m-1}$ where $\mathrm{t}$ acts trivially so that $H_{1}\left(W_{\psi}\right)$ has rank 0 as a $\mathbb{Z}\left[t^{ \pm 1}\right]$-module. $H_{2}\left(W_{\psi}, X_{\phi}\right) \cong\left(\mathbb{Z}\left[t^{ \pm 1}\right]\right)^{\left(\begin{array}{c}m \\ 2\end{array}\right)}$ generated by the core of each $2-$ handle (extended by $\bar{\mu}_{i j} \times I$ ) attached to $X$. Therefore, $\operatorname{Im} \partial_{*}$ is generated by a lift of $\bar{\mu}_{i j}$ in $H_{1}\left(X_{\phi}\right)$ for all $1 \leq i<j \leq m$. To show that $H_{1}\left(X_{\phi}\right)$ has rank 0 it suffices to show that each of the $\bar{\mu}_{i j}$ are $\mathbb{Z}\left[t^{ \pm 1}\right]$-torsion in $H_{1}\left(X_{\phi}\right)$.

Let $F=\left\langle\bar{x}_{1}, \ldots, \bar{x}_{m}\right\rangle$ be the free group of rank $m$ and $f: F \rightarrow G$ be defined by $f\left(\bar{x}_{i}\right)=\bar{x}_{i}$. We have the following $\left(\begin{array}{c}m \\ 3\end{array}\right)$ Jacobi relations in $F / F^{\prime \prime}[4$, Proposition 7.3.6]. For all $1 \leq i<j<k \leq m$,

$$
\left[\bar{x}_{i},\left[\bar{x}_{j}, \bar{x}_{k}\right]\right]\left[\bar{x}_{j},\left[\bar{x}_{k}, \bar{x}_{i}\right]\right]\left[\bar{x}_{k},\left[\bar{x}_{i}, \bar{x}_{j}\right]\right]=1 \bmod F^{\prime \prime} .
$$

Using $f$, we see that these relations hold in $G / G^{\prime \prime}$ as well. From (5), we can write

$$
\left[\bar{x}_{i}, \bar{x}_{j}\right]=\left[\left[v_{i j}, \bar{x}_{j}\right], \bar{x}_{i}\right] \bar{\mu}_{i j} \bmod G^{\prime \prime} .
$$

Hence for each $1 \leq i<j<k \leq m$ we have the Jacobi relation $J(i, j, k)$ in $G / G^{\prime \prime}$

$$
\begin{aligned}
1= & {\left[\bar{x}_{i},\left[\bar{x}_{j}, \bar{x}_{k}\right]\right]\left[\bar{x}_{j},\left[\bar{x}_{i}, \bar{x}_{k}\right]^{-1}\right]\left[\bar{x}_{k},\left[\bar{x}_{i}, \bar{x}_{j}\right]\right] \bmod G^{\prime \prime} } \\
= & {\left[\bar{x}_{i},\left[\left[v_{j k}, \bar{x}_{k}\right], \bar{x}_{j}\right] \bar{\mu}_{j k}\right]\left[\bar{x}_{j}, \bar{\mu}_{i k}^{-1}\left[\bar{x}_{i},\left[v_{i k}, \bar{x}_{k}\right]\right]\right] } \\
& {\left[\bar{x}_{k},\left[\left[v_{i j}, \bar{x}_{j}\right], \bar{x}_{i}\right] \bar{\mu}_{i j}\right] \bmod G^{\prime \prime} } \\
= & {\left[\bar{x}_{i},\left[\left[v_{j k}, \bar{x}_{k}\right], \bar{x}_{j}\right]\right]\left[\bar{x}_{i}, \bar{\mu}_{j k}\right]\left[\bar{x}_{j}, \bar{\mu}_{i k}^{-1}\right]\left[\bar{x}_{j},\left[\bar{x}_{i},\left[v_{i k}, \bar{x}_{k}\right]\right]\right] } \\
& {\left[\bar{x}_{k},\left[\left[v_{i j}, \bar{x}_{j}\right], \bar{x}_{i}\right]\right]\left[\bar{x}_{k}, \bar{\mu}_{i j}\right] \bmod G^{\prime \prime} } \\
= & {\left[\bar{x}_{i}, \bar{\mu}_{j k}\right]\left[\bar{x}_{j}, \bar{\mu}_{i k}^{-1}\right]\left[\bar{x}_{k}, \bar{\mu}_{i j}\right]\left[\bar{x}_{i},\left[\left[v_{j k}, \bar{x}_{k}\right], \bar{x}_{j}\right]\right]\left[\bar{x}_{j},\left[\bar{x}_{i},\left[v_{i k}, \bar{x}_{k}\right]\right]\right] } \\
& {\left[\bar{x}_{k},\left[\left[v_{i j}, \bar{x}_{j}\right], \bar{x}_{i}\right]\right] \bmod G^{\prime \prime} . }
\end{aligned}
$$

Moreover, for each component of the trivial link $L_{i}$ the longitude, $l_{i}$, of $L_{i}$ is trivial in $G$ and is a product of commutators of $\bar{\mu}_{i j}$ with a conjugate of $\bar{x}_{j}$. We 
can write each of the longitudes (see Figure 4) as

$$
\begin{aligned}
l_{i}= & \prod_{j<i} \alpha_{j} \lambda_{j}^{-1} \bar{\mu}_{j i}^{-1} \lambda_{j} \cdot \prod_{k>i} \bar{\mu}_{i k} \beta_{k} \bmod G^{\prime \prime} \\
= & \prod_{j<i}\left(\lambda_{j}^{-1} x_{j n_{j i}}^{-1} \bar{\mu}_{j i} x_{j n_{j i}} \lambda_{j}\right) \lambda_{j}^{-1} \bar{\mu}_{j i}^{-1} \lambda_{j} \cdot \\
& \prod_{k>i} \bar{\mu}_{i k}\left(\lambda_{k} x_{k n_{k i}}^{-1} \lambda_{k}^{-1} \bar{\mu}_{i k}^{-1} \lambda_{k} x_{k n_{k i}} \lambda_{k}^{-1}\right) \\
= & \prod_{j<i}\left[x_{j n_{j i}}^{-1}, \bar{\mu}_{j i}\right]^{\lambda_{j}^{-1}} \cdot \prod_{k>i}\left[\bar{\mu}_{i k}, \lambda_{k} x_{k n_{k i}}^{-1} \lambda_{k}^{-1}\right] \\
= & \prod_{j<i}\left[\bar{x}_{j}^{-1}, \bar{\mu}_{j i}\right] \cdot \prod_{k>i}\left[\bar{\mu}_{i k}, \bar{x}_{k}^{-1}\right] \bmod G^{\prime \prime} .
\end{aligned}
$$

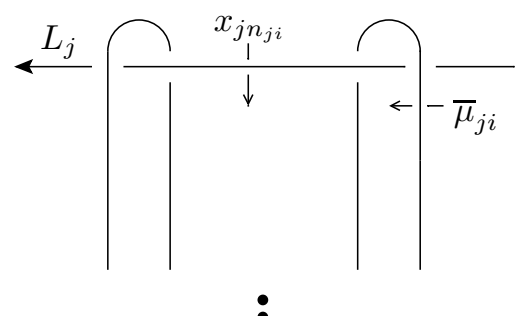

$$
\text { ! }
$$
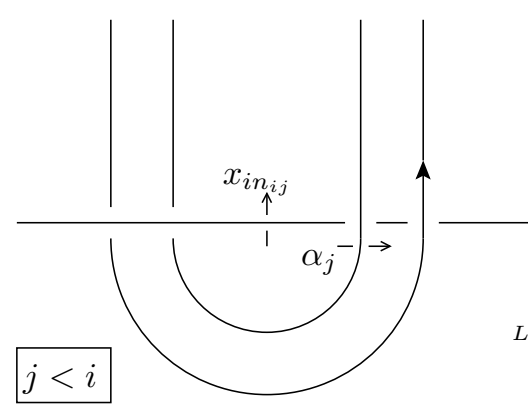

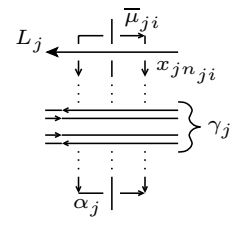

Figure 4

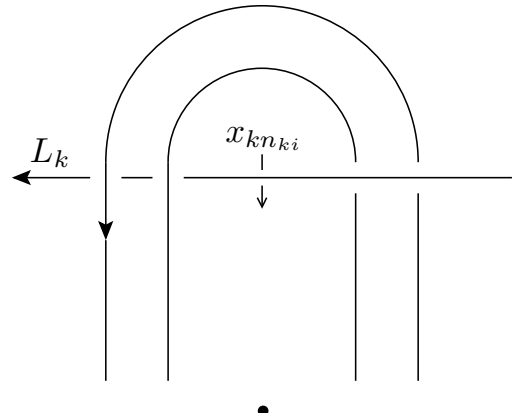

$$
\text { : }
$$

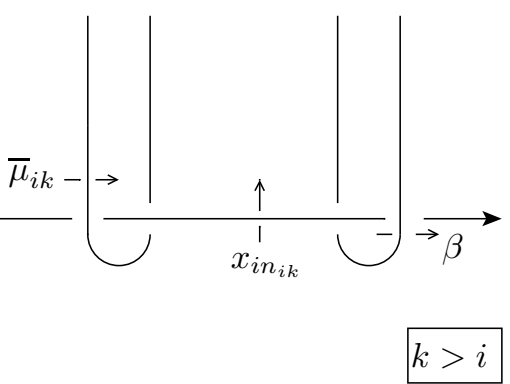

$k>i$ 
It follows that

$$
\prod_{j<i}\left[\bar{x}_{j}^{-1}, \bar{\mu}_{j i}\right] \cdot \prod_{k>i}\left[\bar{\mu}_{i k}, \bar{x}_{k}^{-1}\right]=1 \bmod G^{\prime \prime} .
$$

Since $G^{\prime \prime} \subset[\operatorname{ker} \phi, \operatorname{ker} \phi]$, the relations in (7) and (8) hold in $H_{1}\left(X_{\phi}\right)$ $(=\operatorname{ker} \phi /[\operatorname{ker} \phi, \operatorname{ker} \phi])$ as well. Suppose $\phi: G \rightarrow \mathbb{Z}$ is defined by sending $\bar{x}_{i} \longmapsto$ $t^{n_{i}}$. Since $\phi$ is surjective, $n_{N} \neq 0$ for some $N$. We consider a subset of $\left(\begin{array}{c}m \\ 2\end{array}\right)$ relations in $H_{1}\left(X_{\phi}\right)$ that we index by $(i, j)$ for $1 \leq i<j \leq m$. When $i=N$ or $j=N$ we consider the $m-1$ relations

$$
\text { (i) } \quad R_{i N}=l_{i} \quad \text { and } \quad \text { (ii) } \quad R_{N j}=l_{j}^{-1} \text {. }
$$

Rewriting $l_{i}$ as an element of the $\mathbb{Z}\left[t^{ \pm 1}\right]$-module $H_{1}\left(X_{\phi}\right)$ generated by $\left\{\bar{\mu}_{i j} \mid 1 \leq i<j \leq m\right\}$ from (8) we have

$$
\begin{aligned}
R_{i N}= & \sum_{j<i}\left(t^{-n_{j}}-1\right) \bar{\mu}_{j i}+\sum_{k>i}\left(1-t^{-n_{k}}\right) \bar{\mu}_{i k} \\
= & \sum_{j<i} t^{-n_{j}}\left(1-t^{n_{j}}\right) \bar{\mu}_{j i}+\sum_{k>i} t^{-n_{k}}\left(t^{n_{k}}-1\right) \bar{\mu}_{i k} \\
= & \sum_{j<i}\left[\left(1-t^{n_{j}}\right)+\left(t^{-n_{j}}-1\right)\left(1-t^{n_{j}}\right)\right] \bar{\mu}_{j i}+ \\
& \sum_{k>i}\left[\left(t^{n_{k}}-1\right)+\left(t^{-n_{k}}-1\right)\left(t^{n_{k}}-1\right)\right] \bar{\mu}_{i k} .
\end{aligned}
$$

Similarily, we have

$$
\begin{aligned}
R_{N j}= & \sum_{i<j}\left[\left(t^{n_{i}}-1\right)+\left(t^{-n_{i}}-1\right)\left(t^{n_{i}}-1\right)\right] \bar{\mu}_{i j}+ \\
& \sum_{k>j}\left[\left(1-t^{n_{k}}\right)+\left(t^{-n_{k}}-1\right)\left(1-t^{n_{k}}\right)\right] \bar{\mu}_{j k} .
\end{aligned}
$$

For the other $\left(\begin{array}{c}m-1 \\ 3\end{array}\right)$ relations, we use the Jacobi relations from (7). Define $R_{i j}$ to be

$$
R_{i j}=\left\{\begin{array}{c}
J(N, i, j) \text { for } N<i<j \\
J(i, N, j)^{-1} \text { for } i<N<j . \\
J(i, j, N) \text { for } i<j<N
\end{array}\right.
$$

We can write these relations as

$$
R_{i j}=\left\{\begin{array}{l}
\left(t^{n_{j}}-1\right) \bar{\mu}_{N i}+\left(1-t^{n_{i}}\right) \bar{\mu}_{N j}+\left(t^{n_{N}}-1\right) \bar{\mu}_{i j}+ \\
\left(t^{n_{N}}-1\right)\left(t^{n_{i}}-1\right)\left(t^{n_{j}}-1\right)\left(\widetilde{v}_{i j}+\widetilde{v}_{N j}-\widetilde{v}_{N j}\right) \text { for } N<i<j \\
\left(1-t^{n_{j}}\right) \bar{\mu}_{i N}+\left(t^{n_{N}}-1\right) \bar{\mu}_{i j}+\left(1-t^{n_{i}}\right) \bar{\mu}_{N j}+ \\
\left(t^{n_{N}}-1\right)\left(t^{n_{i}}-1\right)\left(t^{n_{j}}-1\right)\left(-\widetilde{v}_{i N}-\widetilde{v}_{N j}+\widetilde{v}_{i j}\right) \text { for } i<N<j \\
\left(t^{n_{N}}-1\right) \bar{\mu}_{i j}+\left(1-t^{n_{j}}\right) \bar{\mu}_{i N}+\left(t^{n_{i}}-1\right) \bar{\mu}_{j N}+ \\
\left(t^{n_{N}}-1\right)\left(t^{n_{i}}-1\right)\left(t^{n_{j}}-1\right)\left(\widetilde{v}_{i j}+\widetilde{v}_{j N}-\widetilde{v}_{i N}\right) \text { for } i<j<N
\end{array}\right.
$$


where $\widetilde{v}_{i j}$ is a lift of $v_{i j}$.

For $1 \leq i<j \leq m$ order the pairs $i j$ by the dictionary ordering. That is, $i j<l k$ provided either $i<l$ or $j<k$ when $i=l$. The relations above give us an $\left(\begin{array}{c}m \\ 2\end{array}\right) \times\left(\begin{array}{c}m \\ 2\end{array}\right)$ matrix $M$ with coefficients in $\mathbb{Z}\left[t^{ \pm 1}\right]$. The $(i j, k l)^{t h}$ component of $M$ is the coefficient of $\bar{\mu}_{k l}$ in $R_{i j}$. We claim for now that

$$
M=\left(t^{n_{N}}-1\right) I+(t-1) S+(t-1)^{2} E
$$

for some "error" matrix $E$ where $I$ is the identity matrix and $S$ is a skewsymmetric matrix. For an example, when $m=4$ and $N=1, M$ is the matrix

$$
\left[\begin{array}{cccccc}
t^{n_{1}}-1 & 0 & 0 & 1-t^{n_{3}} & 1-t^{n_{4}} & 0 \\
0 & t^{n_{1}}-1 & 0 & t^{n_{2}}-1 & 0 & 1-t^{n_{4}} \\
0 & 0 & t^{n_{1}}-1 & 0 & t^{n_{2}}-1 & t^{n_{3}}-1 \\
t^{n_{3}}-1 & 1-t^{n_{2}} & 0 & t^{n_{1}}-1 & 0 & 0 \\
t^{n_{4}}-1 & 0 & 1-t^{n_{2}} & 0 & t^{n_{1}}-1 & 0 \\
0 & t^{n_{4}}-1 & 1-t^{n_{3}} & 0 & 0 & t^{n_{1}}-1
\end{array}\right]+(t-1)^{2} E .
$$

The proof of (12) is left until the end.

We will show that $M$ is non-singular as a matrix over the quotient field $\mathbb{Q}(t)$. Consider the matrix $A=\frac{1}{t-1} M$. We note that $A$ is a matrix with entries in $\mathbb{Z}\left[t^{ \pm 1}\right]$ and $A(1)$ evaluated at $t=1$ is

$$
A(1)=N I+S(1) \text {. }
$$

To show that $M$ is non-singular, it suffices to show that $A(1)$ is non-singular.

Consider the quadratic form $q: \mathbb{Q}^{\left(\begin{array}{c}m \\ 2\end{array}\right)} \rightarrow \mathbb{Q}^{\left(\begin{array}{c}m \\ 2\end{array}\right)}$ defined by $q(z) \equiv z^{T} A(1) z$ where $z^{T}$ is the transpose of $z$. Since $A(1)=N I+S(1)$ where $S(1)$ is skew-symmetric we have,

$$
q(z)=N \sum z_{i}^{2}
$$

Moreover, $N \neq 0$ so $q(z)=0$ if and only if $z=0$. Let $z$ be a vector satisfying $A(1) z=0$. We have $q(z)=z^{T} A(1) z=z^{T} 0=0$ which implies that $z=0$. Therefore $M$ is a non-singular matrix. This implies that each element $\bar{\mu}_{i j}$ is $\mathbb{Z}\left[t^{ \pm 1}\right]$-torsion which will complete the the proof once we have established the above claim.

We ignore entries in $M$ that lie in $J^{2}$ where $J$ is the augmentation ideal of $\mathbb{Z}\left[t^{ \pm 1}\right]$ since they only contribute to the error matrix $E$. Using (9), (10), and (11) above we can explicitely write the entries in $M\left(\bmod J^{2}\right)$. Let $m_{i j, l k}$ denote the $(i j, l k)$ entry of $M\left(\bmod J^{2}\right)$. 
Case $1(j=N)$ : From (9) we have

$$
m_{i N, l i}=1-t^{n_{l}}, m_{i N, i k}=t^{n_{k}}-1,
$$

and $m_{i N, l k}=0$ when neither $l$ nor $k$ is equal to $N$.

Case $2(i=N)$ : From (10) we have

$$
m_{N j, l j}=t^{n_{l}}-1, m_{N j, j k}=1-t^{n_{k}},
$$

and $m_{N j, l k}=0$ when neither $l$ nor $k$ is equal to $N$.

Case $3(N<i<j)$ : From (11) we have

$$
m_{i j, N i}=t^{n_{j}}-1, m_{i j, N j}=1-t^{n_{i}}, m_{i j, i j}=t^{n_{N}}-1,
$$

and $m_{i j, l k}=0$ otherwise.

Case $4(i<N<j)$ : From (11) we have

$$
m_{i j, i N}=1-t^{n_{j}}, m_{i j, i j}=t^{n_{N}}-1, m_{i j, N j}=1-t^{n_{i}},
$$

and $m_{i j, l k}=0$ otherwise.

Case $5(i<j<N)$ : From (11) we have

$$
m_{i j, i j}=t^{n_{N}}-1, m_{i j, i N}=1-t^{n_{j}}, m_{i j, j N}=t^{n_{i}}-1,
$$

and $m_{i j, l k}=0$ otherwise.

We first note that in each of the cases, the diagonal entries $m_{i j, i j}$ are all $t^{n_{N}}-1$. Next, we will show that the off diagonal entries have the property that $m_{i j, l k}=$ $-m_{l k, i j}$ for $i j<l k$. This will complete the proof of the claim since we see that each entry is divisible by $t-1$.

We verify the skew symmetry in Cases 1 and 3 . The other cases are similar and we leave the verifications to the reader.

Case $1(j=N)$ :

$$
m_{i N, l i}=1-t^{n_{l}}=-m_{l i, i N}(\text { case } 5)
$$

and

$$
m_{i N, i k}=t^{n_{k}}-1=-m_{i k, i N}(\text { case } 4) .
$$

Case $3(N<i<j)$ :

$$
m_{i j, N i}=t^{n_{j}}-1=-m_{N i, i j}(\text { case } 2)
$$

and

$$
m_{i j, N j}=1-t^{n_{i}}=-m_{N j, i j}(\text { case } 2) .
$$

Geometry ${ }^{6}$ Topology, Volume 6 (2002) 
Proposition 3.3 Let $X$ be as in Theorem 3.1, $G=\pi_{1}(X)$ and $F$ be the free group on 2 generators. There is no epimorphism from $G$ onto $F / F_{4}$.

Proof Let $F=\langle x, y\rangle$ be the free group and $\phi: F / F_{4} \rightarrow\langle t\rangle$ be defined by $x \longmapsto t$ and $y \longmapsto 1$. Suppose that there exists a surjective map $\eta: G \rightarrow F / F_{4}$. Let $N=\operatorname{ker} \phi$ and $H=\operatorname{ker}(\eta \circ \phi)$. Since $\eta$ is surjective we get an epimorphism of $\mathbb{Z}\left[t^{ \pm 1}\right]$-modules $\widetilde{\eta}: H / H^{\prime} \rightarrow N / N^{\prime}$. From (6) we get the short exact sequence

$$
0 \rightarrow \operatorname{Im} \partial_{*} \stackrel{i}{\rightarrow} H_{1}\left(X_{\eta \circ \phi}\right) \rightarrow H_{1}\left(W_{\psi}\right) \rightarrow 0 .
$$

Let $J$ be the augmentation ideal of $\mathbb{Z}\left[t^{ \pm 1}\right]$. We compute $N / N^{\prime} \cong \mathbb{Z}\left[t^{ \pm 1}\right] / J^{3}$ so that $\widetilde{\eta}: H_{1}\left(X_{\eta \circ \phi}\right) \rightarrow \mathbb{Z}\left[t^{ \pm 1}\right] / J^{3}$. Let $\sigma \in H_{1}\left(X_{\eta \circ \phi}\right)$ such that $\widetilde{\eta}(\sigma)=1$. Since every element in $H_{1}\left(W_{\psi}\right) \cong \bigoplus_{i=1}^{m-1} \frac{\mathbb{Z}\left[t^{ \pm 1}\right]}{J}$ is $(t-1)$-torsion, $(t-1) \sigma \in$ $\operatorname{Im} \partial_{*}$ hence $t-1 \in \operatorname{Im}(\widetilde{\eta} \circ i)$. Recall that in the proof of the Theorem 3.1, we showed that there exists a surjective $\mathbb{Z}\left[t^{ \pm 1}\right]$-module homomorphism $\rho: P \rightarrow$ $\operatorname{Im} \partial_{*} \quad$ where $P$ is finitely presented as

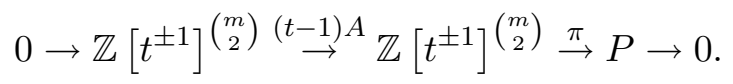

Let $g: P \rightarrow \mathbb{Z}\left[t^{ \pm 1}\right] / J^{3}$ defined by $g \equiv \widetilde{\eta} \circ i \circ \rho$. Since $\rho$ is surjective, $t-1 \in$ $\operatorname{Im} g$. After tensoring with $\mathbb{Q}\left[t^{ \pm 1}\right]$, we get a map $g: P \otimes_{\mathbb{Z}\left[t^{ \pm 1}\right]} \mathbb{Q}\left[t^{ \pm 1}\right] \rightarrow$ $\mathbb{Q}\left[t^{ \pm 1}\right] / J^{3}$. It is easy to see that either $g$ is surjective or the image of $g$ is the submodule generated by $t-1$. Note that the submodule generated by $t-1$ is isomorphic $\mathbb{Q}\left[t^{ \pm 1}\right] / J^{2}$. Hence, in either case, we get a surjective map $h: P \otimes_{\mathbb{Z}\left[t^{ \pm 1}\right]} \mathbb{Q}\left[t^{ \pm 1}\right] \rightarrow \mathbb{Q}\left[t^{ \pm 1}\right] / J^{2}$.

Consider the $\mathbb{Q}\left[t^{ \pm 1}\right]$-module $P^{\prime}$ presented by $A$. Let $h^{\prime}: \mathbb{Q}\left[t^{ \pm 1}\right]^{\left(\begin{array}{c}m \\ 2\end{array}\right)} \rightarrow$ $\mathbb{Q}\left[t^{ \pm 1}\right] / J^{2}$ be defined by $h^{\prime}=(t-1) h \circ \pi$. Since

$$
h^{\prime}(A(\sigma))=(t-1) h(\pi(A(\sigma)))=h(\pi((t-1) A(\sigma)))=h(0)=0,
$$

this defines a map $h^{\prime}: P^{\prime} \rightarrow \mathbb{Q}\left[t^{ \pm 1}\right] / J^{2}$ whose image is the submodule generated by $t-1$. It follows that $P^{\prime}$ maps onto $\mathbb{Q}\left[t^{ \pm 1}\right] / J$. Setting $t=1$, the vector space over $\mathbb{Q}$ presented by $A(1)$ maps onto $\mathbb{Q}$. Therefore $\operatorname{det}(A(1))=0$. However, it was previously shown that $A(1)$ was non-singular which is a contradiction.

Corollary 3.4 For any closed, orientable 3-manifold $Y$ with $P / P_{4} \cong G / G_{4}$ where $P=\pi_{1}(Y)$ and $G=\pi_{1}(X)$ is the fundamental group of the examples in Theorem 3.1, $c(Y)=1$. 
Using Proposition 3.3, it is much easier to show that there exist hyperbolic 3 -manifolds with cut number 1.

Corollary 3.5 For each $m \geq 1$ there exist closed, orientable, hyperbolic 3manifolds $Y$ with $\beta_{1}(Y)=m$ such that $\pi_{1}(Y)$ cannot map onto $F / F_{4}$ where $F$ is the free group on 2 generators.

Proof Let $X$ be one of the 3-manifolds in Theorem 3.1. By [7, Theorem 2.6], there exists a degree one map $f: Y \rightarrow X$ where $Y$ is hyperbolic and $f_{*}$ is an isomorphism on $H_{*}$. Denote by $G=\pi_{1}(X)$ and $P=\pi_{1}(Y)$. It follows from Stalling's theorem [9] that $f$ induces an isomorphism $f_{*}: P / P_{n} \rightarrow G / G_{n}$. In particular this is true for $n=4$ which completes the proof.

\section{References}

[1] T Cochran, P Melvin, Quantum cylotomic orders of 3-manifolds, Topology 40 (2001) 95-125

[2] P Gilmer, T Kerler, Cut numbers of 3-manifolds via quantum orders in $\mathbb{Z}\left[\zeta_{5}\right]$, preprint

[3] J Howie, Free subgroups in groups of small deficiency, J. Group Theory 1 (1998) 95-112

[4] A Kawauchi, A survey of knot theory, Birkhauser Verlag, Basel (1996)

[5] T Kerler, Homology TQFTs and the Alexander-Reidemeister invariant of 3manifolds via Hopf algebras and skein theory, preprint

[6] C Leininger, A Reid, The co-rank conjecture for 3-manifold groups, Algebr. Geom. Topol. 2 (2002) 37-50

[7] D Ruberman, Seifert surfaces of knots in $S^{4}$, Pacific J. Math. 145 (1990)

[8] A S Sikora, Cut numbers of 3-manifolds, preprint

[9] J Stallings, Homology and central series of groups, J. Algebra 2 (1965) 170-181

[10] W P Thurston, A norm for the homology of 3-manifolds, Mem. Amer. Math. Soc. 59 (1986) i-vi, 99-130 